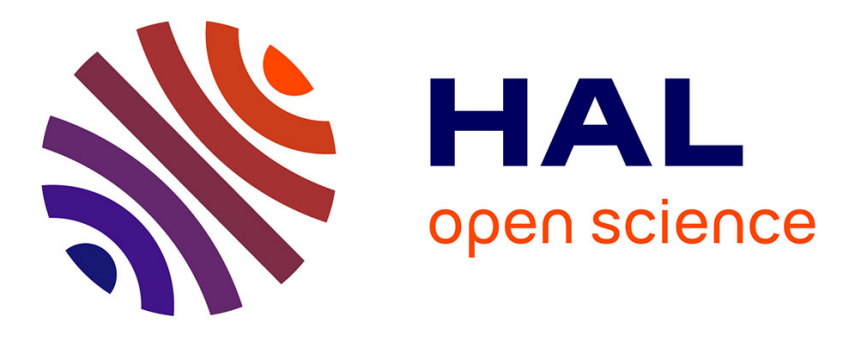

\title{
Application of DAFS Spectroscopy to Study the Variations of Fe local structure in a Fe/Ir(100) Superlattice
}

Hubert Renevier, J. Hodeau, V. Dalakas, P. Wolfers, J. Bérar, S. Andrieu, J. Weigelt, R. Frahm

\section{To cite this version:}

Hubert Renevier, J. Hodeau, V. Dalakas, P. Wolfers, J. Bérar, et al.. Application of DAFS Spectroscopy to Study the Variations of Fe local structure in a Fe/ $\operatorname{Ir}(100)$ Superlattice. Journal de Physique IV Proceedings, 1997, 7 (C2), pp.C2-741-C2-743. 10.1051/jp4:1997223 . jpa-00255302

\section{HAL Id: jpa-00255302 https://hal.science/jpa-00255302}

Submitted on 1 Jan 1997

HAL is a multi-disciplinary open access archive for the deposit and dissemination of scientific research documents, whether they are published or not. The documents may come from teaching and research institutions in France or abroad, or from public or private research centers.
L'archive ouverte pluridisciplinaire HAL, est destinée au dépôt et à la diffusion de documents scientifiques de niveau recherche, publiés ou non, émanant des établissements d'enseignement et de recherche français ou étrangers, des laboratoires publics ou privés. 


\title{
Application of DAFS Spectroscopy to Study the Variations of Fe Local Structure in a Fe/Ir(100) Superlattice
}

\author{
H. Renevier***, J.L. Hodeau*****, V. Dalakas*, P. Wolfers*, J.F. Bérar*, S. Andrieu****, \\ J. Weigelt***** and R. Frahm*****
}

\author{
* Laboratoire de Cristallographie, CNRS, BP. 166, 38042 Grenoble cedex 09, France \\ ** Université Joseph Fourier, BP. 53, 38041 Grenoble cedex 09, France \\ *** ESRF, BP. 220, 38043 Grenoble cedex 09, France \\ **** LMPSM, URA 155, Université de Nancy I, BP. 239, 54506 Vandoeuvre, Nancy cedex, France \\ ***** HASYLAB, Notkestr. 85, 22607 Hamburg, Germany
}

\begin{abstract}
Superlattice studies are important to synthesise $3 d$ metals in new crystalline structure which may exhibit exotic magnetic properties. The challenge is to relate these properties to the details of the chemical gradient and the local strain. We report here on the use of Diffraction Anomalous Fine Structure (DAFS) spectroscopy to separate the XAFS-like information about $\mathrm{Fe}$ atoms located at different crystallographic sites of an $\mathrm{Fe} / \mathrm{Ir}(100)$ superlattice. We obtained the $\mathrm{Fe}$ nearest neighbor distances at the Ir-Fe interfaces by the use of a new crystallographic-based analysis of the DAFS data.
\end{abstract}

\section{INTRODUCTION}

Diffraction Anomalous Fine Structure (DAFS) spectroscopy provides a site-selective XAFS-like information about the local atomic enviromment and the chemical state of the 'edge atom' through resonant X-ray scattering and diffraction process. Several years after a few pioneering works [1], Stragier et al. [2] presented an elegant demonstration of this method on a copper single crystal and later, several groups applied it on different types of samples like thin films, superlattices [3, 4], powders [5], single crystals [6]. In principle, DAFS provides site selective spectroscopic information, however most results were obtained with materials for which structural information could be extracted with single site-selective Bragg reflections. Site selective information with non "academic" samples was successfully extracted in the DANES region (Diffraction Near Edge Structure) of the spectrum, however due to insufficient statistics no information in the EDAFS region (Extended Diffraction Anomalous Fine Structure) could be obtained [6]. Regarding superlattices, only spatial selectivity of DAFS has been used. The first DAFS experiments on several pseudomorphic Fe/Ir(100) superlattices were carried out at the Ir edge to recover the Ir crystallographic parameters in the buffer and the superlattice, separately [4] (spatial selectivity). We present here a crystallographic-based site-selective EDAFS study of an $\mathrm{Fe} / \mathrm{Ir}(100)$ superlattice. Fe/Ir superlattices were synthesized to stabilize Fe in new crystalline structures with the hope of obtaining new magnetic properties. To relate these properties to the Fe local structure, superlattices are well suited two-dimensional systems. Different strained Fe/Ir structures may be synthesized by varying the relative $\mathrm{Fe}$ and Ir layer thicknesses [7]. It has been shown that Fe relaxes into a body centered cubic structure ("bcc phase" as bulk Fe) in thick Fe layers (more than 10 atomic planes) when grown on three Ir planes at most, whereas in thin Fe layers (less than 5 atomic planes) Fe has a body centered tetragonal structure (bct) with a c/a ratio near 1.25 [8] (pseudomorphic regime). For "intermediate thicknesses" with thick Fe and Ir layers (more than five atomic planes each) the Fe structure is still not accurately known.

\section{EXPERIMENTS}

The $\mathrm{Fe} / \mathrm{Ir}$ superlattice studied was grown by molecular beam epitaxy on an (100) $\mathrm{MgO}$ substrate, without an Ir buffer, and made of $80 \mathrm{Fe} / \mathrm{Ir}$ bilayers of total thickness of $0.24 \mu \mathrm{m}$. The diffraction spectra showed several well defined superlattice $00 \mathrm{l}$ reflections indicating a good epitaxy. The DAFS spectra were recorded at the X-ray undulator Beamline BWI at HASYLAB [9]. The sample was mounted on a diffractometer and the intensities were recorded in reflection mode, with the polarisation perpendicular to the plane of diffraction. A complete diffraction spectra was collected at an energy close to the Ir LIII-edge $(11000 \mathrm{eV})$ at the beamline D2AM at the ESRF, and two diffraction spectra of reflections close to the first order intereticular distance $(\mathrm{d} \approx 1.5 \AA)$ were collected at HASYLAB at energies above and below the Fe K edge $(6900 \mathrm{eV}$ and $7300 \mathrm{eV})$. Since the shape of the superlattice reflections was well defined with a large FWHM $\left(0.5^{\circ}\right)$, we used a top-DAFS-scan procedure for collecting the DAFS spectra, i.e, we measured the diffracted intensity at the top of the reflections while scanning the beam energy. DAFS spectra of five reflections (from 0036 to 0044 ) were collected at the Fe K-edge with a varying step (down to $2 \mathrm{eV}$ ) in the $6900-7800 \mathrm{eV}$ range. On the two beamlines BW1 and D2AM, the top-DAFS-scan method records a complete DAFS spectrum in less than two hours. Due to the mosaïcity, inhomogenous absorption, or narrow FWHM, this procedure can not be applied to all samples [6], but is well suited for superlattices, monolayers or thin films. The fluorescence signal was simultaneously recorded for energy calibration and for absorption correction. To correct the diffracted signal for the fluorescence of the sample, the background on each side of the reflection was also measured. 


\section{EDAFS ANALYSIS}

Changes in the diffracted intensities near an absorption edge are related to those of all anomalous atomic scattering factors. In the forward scattering limit the atomic scattering factor of an atom A on site $j$, may be split above the edge into smooth and oscillatory parts $[1 b, 2,3]$ :

$$
f_{A j}(\vec{Q}, E)=f_{O A}(\vec{Q})+f_{O A}^{\prime}(E)+i f_{O A}^{\prime \prime}(E)+f_{O A}^{\prime \prime}(E)\left[\chi_{A j}^{\prime}+i \chi_{A j}^{\prime \prime}\right]
$$

where $f_{O A}$ is the Thomson scattering, $f_{O A}^{\prime}$ and $f_{O A}^{\prime \prime}$ are the "bare" atom resonant and anti-resonant corrections to $f_{A j}$, respectively. $\vec{Q}$ is the scattering vector and $\mathrm{E}$ the energy of the incident beam ; $\chi_{A j}^{\prime}+i \chi_{A j}^{\prime \prime}$ is the complex fine structure, $\chi_{A j}^{\prime \prime}$ is equivalent to the EXAFS signal and $\chi_{A j}^{\prime}$ is related to $\chi_{A j}^{\prime \prime}$ via the Kramers-Kronig transform. The oscillatory part of the DAFS spectra $\left(\chi_{\bar{Q}}(E)\right)$ were extracted and normalized according to the following formula for a centrosymmetric cell :

$$
\chi_{\bar{Q}}(E)=\frac{I}{\alpha} \sum_{j} \alpha_{A j}\left(\chi_{A j}^{\prime}+\varepsilon \chi_{A j}^{\prime \prime}\right)=\left(\frac{I_{u c}-I_{a c 0}}{I_{a c 0}}\right) \frac{\left\|F_{0}\right\|^{2}}{2 \alpha \operatorname{Re}\left(F_{0}\right) \Delta\left(f_{o A}^{\prime \prime}\right)}
$$

where $\varepsilon_{\overline{\boldsymbol{Q}}}(E)=\operatorname{Im}\left(F_{0}\right) / \operatorname{Re}\left(F_{0}\right), \alpha_{A j} / \alpha$ are the weights of the different anomalous sites to the diffracted intensities, $\alpha$ is the sum all $\alpha_{A j}, I_{a c}$ is the measured intensity corrected for absorption and $I_{a c o}$ the smooth part of $I_{a c}, \Delta\left(f_{O A}^{\prime \prime}\right)$ is the jump of $f_{O A}^{\prime \prime}$ beyond the edge and $\mathrm{F}_{0}$ the smooth part of the structure factor. The next step is to solve for $\chi_{A j}^{\prime}$ and $\chi_{A j}^{\prime \prime}$ a linear system of $\mathrm{M}$ equations (2) and $2 \mathrm{~N}$ unknowns for each energy ; where $\mathrm{M}$ is the number of $\chi_{\bar{Q}}(E)$ EDAFS spectra and $\mathrm{N}$ the number of inequivalent anomalous site $j$. The Kramers-Kronig relation between $\chi_{A j}^{\prime}$ and $\chi_{A j}^{\prime \prime}$ is used as an additional constraint.

\section{RESULTS}

The crystallographic structure has been refined to accurately correct for absorption the DAFS spectra and for separating the site dependent XAFS-like information, i.e. obtaining accurate $\alpha_{A j}$. A centered tetragonal centrosymmetric cell has been used with the $c$ axis along the direction of growth, the space group was $14 / \mathrm{m}$. In such a description the superlattice cell along the $\mathrm{c}$ axis is made of two Fe/Ir bilayers and only the $00 l$ reflections with $l=2 \mathrm{n}$ exist. The superlattice periodicity $\mathrm{c}$ was found to be $2 \times 30.989 \AA$ and made of 34 atomic planes filled with $\mathrm{Fe}$ atoms and 8 containing $30 \%$ Ir atoms. Results of the crystallographic refinements show that for this sample which contains only a few Ir atoms, no sharp interfaces exist in between Iridium and Iron layers. The Ir rich planes (hereafter called site Fe11) are filled with $42 \%$ of Ir atoms and the others IrFe mixed planes (site Fe10) with only $17 \%$. In table 1 are reported the relative contribution $\left(\alpha_{\mathrm{Fe} 11} / \alpha\right)$ of $\mathrm{Fe}$ on site Fe11 (the contribution of all other Fe site is $\alpha_{F e l-10} / \alpha=I-\alpha_{F e l l} / \alpha$ ). As we expect, Fe layers mainly contribute to the Bragg peak which correspond to an interplanar distance close to the bcc Fe $\mathrm{d}_{002}\left(\mathrm{~d}_{002}=1.43 \AA\right)$, whereas the Ir rich planes contribute to the small reflections 0036 and 0038 , which therefore contain the detail of the structure at the interfaces. Of all reflections, the 0036 is the most sensitive to the Ir rich planes. This clearly demonstrates the interest for using DAFS spectra to refine the crystallographic structure : the smooth behavior of the DAFS spectra gives information on the net phase $(\alpha)$ of all anomalous sites contributing to the reflection, i.e. on the positions, the Debye-Waller and the occupation factors.

\begin{tabular}{|c|c|c|c|}
\hline 001 & $\alpha$ & $\alpha_{F e 11} / \alpha$ & $d_{001}(\AA)$ \\
\hline 0036 & -1.50 & 1.32 & 1.722 \\
\hline 0038 & -4.06 & 0.50 & 1.631 \\
\hline 0040 & -9.56 & 0.20 & 1.549 \\
\hline 0042 & -28.84 & 0.06 & 1.476 \\
\hline 0044 & 21.36 & -0.08 & 1.409 \\
\hline
\end{tabular}

Table I. The net phase of all anomalous $\mathrm{Fe}$ atoms $(\alpha)$ and the relative contributions of site Fe11 at the interfaces to the Bragg reflections $\left(\alpha_{\mathrm{Fe} 11} / \alpha\right)$.

Figure 1 shows the five EDAFS oscillation spectra normalized according to eq. (2). On going from 0044 to 0036 , one observes a continuous change of the spectra showing the existence of different Fe local structures. Although, the 0036 EDAFS corresponds to a weak signal (less than $8 / 1000$ in absolute value), the signal to noise ratio is satisfactory. We solved the linear system of eq. (2) for the Ir rich $\chi_{F e 11}^{\prime}\left(\& \chi_{F e 11}^{\prime \prime}\right)$ and bulk Fe $\chi_{F e 1-10}^{\prime}\left(\& \chi_{F e l-10}^{\prime \prime}\right)$ fine structures. Figure 2 shows the Fourier transform of both signals $k^{2} \chi_{\mathrm{Fell}}^{\prime \prime}$ and $k^{2} \chi_{\mathrm{Fel-10}}^{\prime \prime}$, plus that of the fluorescence EXAFS $\left(k^{2} \chi_{f \text { fuo }}^{\prime \prime}\right)$. The range for the Fourier transform was from $\mathrm{k}=2.5 \AA^{-1}$ and $12.5 \AA^{-1}$. As expected the Fourier transform of $\chi_{\mathrm{Fe} 1-10}^{\prime \prime}$ and $\chi_{f(k,}^{\prime \prime}$ are very similar, whereas the Fe local environment is quite different at the interfaces. For bcc Fe, the two first nearest neighbor shells have close distances $\left(N_{F e}^{I}=8\right.$ neighbors at $2.48 \AA$ and $N_{F e}^{2}=6$ at $2.86 \AA$ ), therefore their contributions to the EDAFS signal are mixed in 
the first peak of the Fourier transform. For Fe at the interfaces the nearest shells are comprised of Fe and Ir atoms. The number of $\mathrm{Fe}$ and $\mathrm{Ir}$ nearest neighbors in those shells were estimated from the crystallographic structure, assuming a random mixing, and by taking into account the XAFS-like polarisation dependence of the EDAFS signal $\left(N_{F e}^{1}=5.2, N_{F e}^{2}=3.5, N_{l r}^{I}=2.1\right.$ and $N_{F e}^{2}=2.5$, respectiveley). The EXAFS phase and amplitude for Fe-Fe were extracted from a Co metal XAFS spectra, whereas these for Fe-Ir were calculated with Feff6.01 [10] using the refined crystallographic structure.

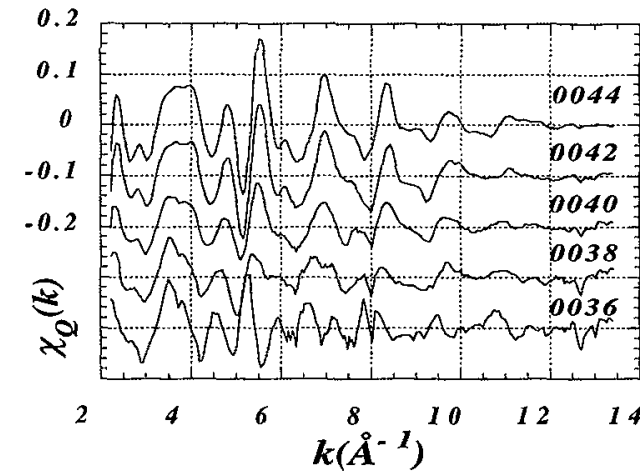

Figure 1: Normalized EDAFS spectra

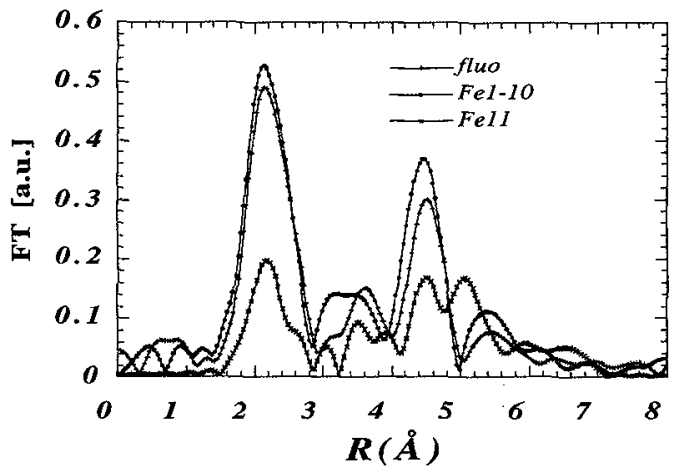

Figure 2: Fourier transforms (see text)

The distances and the Debye-Waller were refined only. The distances corresponding to the sarne shell were initially forced to be equal. The same thing was done for the Debye-Waller. At last, Fe-Fe and Fe-Ir distances for a given shell were found to be very similar. From the back Fourier filtered first nearest neighbor contribution to $\chi_{F e 1 I}^{\prime \prime}$ the averaged in-plane first nearest neighbor distance at the interface, was found to be $2.89 \AA$, i.e. larger than the bulk "bcc Fe" obtained from the back Fourier filtered first nearest neighbor contribution to $\chi_{F e I-10}^{\prime \prime}$ or $\chi_{\text {fluo }}^{\prime \prime}(2.83 \AA)$. Note that $2.83 \AA$ is a slightiy lower value than that of the corresponding distance in bcc Fe metal $(2.86 \AA)$. The average value of the first nearest neighbor distance was found to be $2.59 \AA$ instead of $2.47 \AA$ for the bulk "bcc Fe". This result well agree with the increase of the interplanar distance at the interfaces $(1.43 \AA$ to $1.62 \AA=(1.64+1.60) / 2)$ shown by the crystallographic refinements and the value of $2.89 \AA$ for the in-plane first nearest neighbor. Note that the drastic change of the first nearest neighbor peak of $\chi_{F e I 1}^{\prime \prime}$ (with respect to the $\chi_{F e l-10}^{\prime \prime}$ one), is to a large extent the result of the increase of the first nearest neighbor $\mathrm{Fe}-\mathrm{Fe}$ (Fe-Ir) distance and the presence of Ir atoms atoms in the nearest shells. The tetragonal distortion at the interface play a secondary role. Although the filtered signals does show the presence of Ir atoms in the two first nearest neighbor shells, Fe-Fe contribution dominates in the available range of $\mathrm{k}\left(2.5 \AA^{-1} \leq \mathrm{k} \leq 12.2 \AA^{-1}\right)$. Therefore for obtaining more details about the Ir shells it would be necessary to measure the EDAFS spectra further in $\mathrm{k}$ (at least up to $16 \AA^{-1}$ ) and/or use Fe K-edge DAFS and Ir LIII-edge EXAFS data in a multirefinement procedure.

In conclusion we have used DAFS spectroscopy for studying the different Fe local structure inside an Fe/Ir(100) superlattice. We have shown for the first time that is possible to probe selectively Fe atoms at the Ir-Fe interfaces (two planes out of twenty one of half of the superlattice centrosymmetric cell) and extract information about the local environment. We have also demonstrated the usefullness of a crystallographic-based analysis which combines the analysis of DAFS and diffraction spectra for merging the most accurate description of both the average and local structure.

\section{Acknowledgments}

The crystallographic analysis was based on DPU (Data Processing Unit), an advanced multi-refinements package (P. Wolfers). For the XAFS-like analysis we used the programs of D. Aberdam and A. Michalowicz.

\section{References}

[1] a) Y. Cauchois \& C. Bonnelle, C. R. Acad. Sci. 242, (1956). b) I. Arcon et al., J. Physique C9, 1105, (1987).

[2] H.J. Stragier et al., Phys. Rev. Lett. 69, 3064, (1992).

[3] H.J. Stragier, PhD Thesis, Univ. of Washington, (1993)

[4] H. Renevier et al, , Physica B 208\& 209, 215, (1995).

[5] I.J. Pickering et al., J. Am. Chem. Soc. 115, 6302, (1993).

[6] J. Vacinova et al., J. Synchrotron Rad. 2, 236, (1995).

[7] For a complete review see : S. Andrieu et al,, Phys. Rev. B 52, 9938, (1995).

[8] S. Andrieu et al., Europhys. Lett. 26, 189, (1994).

[9] R. Frahm et al., Rev. Sci. Instrum., in press, (1994).

[10] J. Mustre de Leon et al., Phys. Rev. B 44, 4146, (1991). S.I. Zabinsky et al., phys. Rev. B 52, 2995, (1995). 